\title{
Hepatic Abscess in Preterm Newborn- A rare entity
}

\author{
Anand $S^{1}$, Chanchlani $R^{2}$, Gupta $V^{3}$, Goyal $S^{4}$, Tiwari $A^{5}$ \\ ${ }^{1}$ Dr Shweta Anand, Associate Professor, Department of Paediatrics, ${ }^{2}$ Dr Roshan Chanchlani, Associate Professor, Department \\ of Surgery, ${ }^{3}$ Dr Vivek Gupta, Assistant Professor, Department of Radiodiagnosis, ${ }^{4}$ Dr Shweta Goyal, Assistant Professor \\ Department of Paediatrics, ${ }^{5}$ Dr Astha Tiwari Assistant Professor, Department of Pediatric. All are affiliated with Chirayu \\ Medical College Bhopal, MP, India
}

Address for correspondence: Dr Shweta Anand, Email: drsa007@yahoo.com

\begin{abstract}
Neonatal liver abscess is rare entity and few cases are reported till date. We report a case of newborn who presented with a abdominal lump and a huge liver abscess. Baby underwent Ultrasonography and CT scan and abscess was aspirated under USG guidance. As there was no resolution patient underwent surgical procedure with insertion of pigtail catheter under anaesthesia in operation theatre. Baby is asymptomatic with no residual abscess on follow- up. The patient was managed well with coordination from Paediatric, Radiology and Paediatric surgery department.
\end{abstract}

Keywords: Neonate, Hepatic Abscess, Preterm, Abscess

\section{Introduction}

In 1836, the first report on liver abscess was published by Bright [1]. Neonatal liver abscess is a rare entity and to our knowledge, fewer than 100 cases have been reported in the literature. We present a case and review of current literature pertaining to the management of neonatal liver abscess.

A premature newborn girl born at 35 weeks of gestation, weighing $2.6 \mathrm{~kg}$ was delivered vaginally to a primigravida mother. Baby was kept in NICU for respiratory distress after birth. On day three, she developed sepsis and severe respiratory distress for which baby was put on mechanical ventilation and umbilical venous catheter (UVC) was inserted. She developed neonatal hyperbilirubinemia (ABO incompatibility) and underwent exchange transfusion. She was extubated on day seven.

Nasogastric feeding started on day eighth and it was increased over the subsequent days but baby developed abdominal distension on day eleven and was referred to our tertiary care hospital for further evaluation and management.

On admission, baby had mild tachypnea and on examination liver was enlarged $10 \mathrm{~cm}$ below costal margin, firm, smooth surface with well defined margins, another localised swelling was palpable over right hypochondrium which was extending upto left hypochondrium and left lumbar region. The lump was firm with smooth surface and irregular margin. Investigation revealed $\mathrm{Hb}-12.1 \mathrm{gm} \%$, TLC-12,200/ $\mathrm{mm}^{3}$, platelet count $1.6 \mathrm{lac} / \mathrm{mm}^{3}$, CRP (positive with increasing titer), peripheral smear revealed normocytic, normochromic Anemia with hemolytic picture and toxic granules. Blood culture showed growth of methicillin sensitive staphylococcal aureus (MSSA). LFT revealed bilirubin $7.3 \mathrm{mg} / \mathrm{dl}$, SGPT was 60IU/L, SGOT, 41IU/L and serum alkaline phosphatase was 218 IU/L. USG showed large left hepatic lobe abscess size $78 \times 41.7 \times 68 \mathrm{~mm}^{3}$, volume $110-150 \mathrm{ml}$ approximately with central liquefaction, (Fig 1) subsequently Contrast enhanced CT scan of abdomen shows hepatomegaly with large multiloculated intercommunicating abscess in left hepatic lobe minimal pleural effusion with segmental atelectasis. (Fig 2)

After taking informed consent USG guided therapeutic percutaneous needle aspiration done under asepsis, around $40 \mathrm{ml}$ thick reddish-brown hazy fluid was aspirated which was sent for investigation showed growth of E. Coli $\left(10^{5}\right.$ $/ \mathrm{ml}$ ) and antibiotic were changed according to culture sensitivity.

Repeat USG done showed no regression of abscess so under anaesthesia Ultrasonographic guided pigtail catheter was introduced by Pediatric surgeon, in Operation theatre. Drainage of pus through catheter was done and subsequently repeat USG showed regression of lesion with volume of only $20 \mathrm{ml}$ with further central liquefaction. Catheter was removed on $5^{\text {th }}$ post operative day and intravenous antibiotics according to culture sensitivity were continued for 2 weeks. Patient responded well and there was complete regression of abscess. 


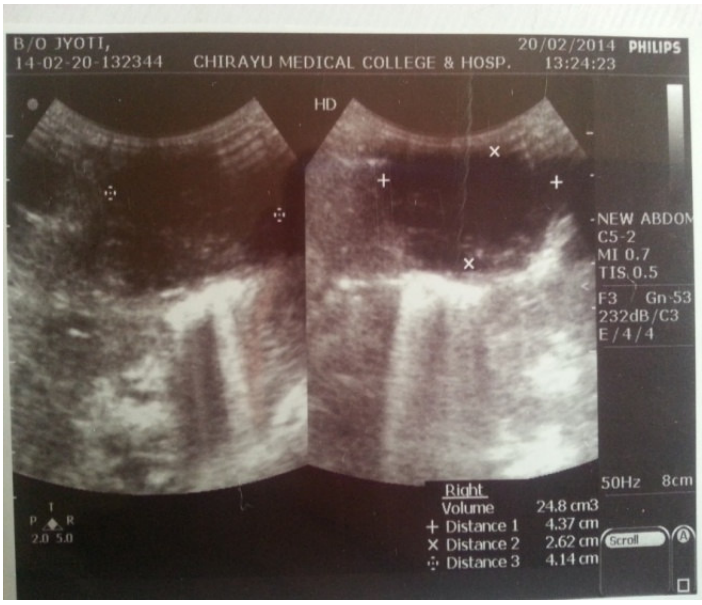

Fig 1: USG Showing abscess with central liquefication

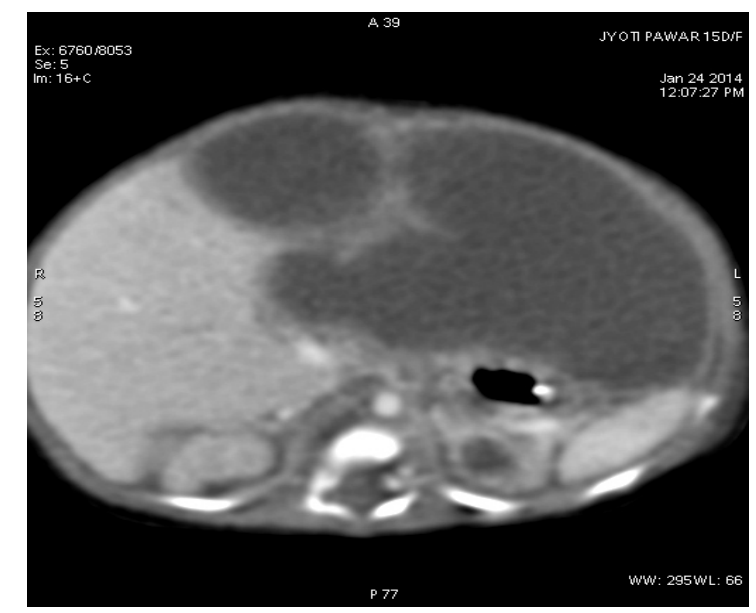

Fig 2: CT Abdomen with Hepatic Abscess

\section{Discussion}

Although, liver abscess has been recognised since the time of Hippocrates but still less than 100 cases has been reported in neonates [2]. The first review of liver abscess appeared in 1936 by Kutsunak who reported 2 infants with fatal peritonitis with solitary liver abscess at necropsy [3].

Neonatal liver abscesses are difficult to diagnose because of rarity, indolent course and lack of suspicion. Incorrect placement of umbilical venous catheter and blood culture proven sepsis are reported to be the most common predisposing factors for liver abscesses followed by central TPN catheter, necrotising enterocolits, surgery and prematurity with no other associated factor[4]. Infection of the liver in the neonate differs from that in the older children in that opportunistic and parasitic infections are unusual.

The classical presentation of liver abscess with fever, hepatomegaly and right upper abdominal pain is not generally present in neonates [5]. As in our case although baby had exchange transfusion but UVC was in correct position so sepsis and prematurity can be the precipitating factor for liver abscess.

The most common organisms are Staphylococcus Aureus, Streptococcus pyogenes and E Coli isolated from solitary hepatic abscess in neonates [6]. In our case, E coli was the causative pathogen.

Abdominal ultrasonography, CT scan, and liver scan with technetium are useful studies to visualise a possible hepatic abscess. Ultrasound is the investigation of choice in pediatric patients for liver abscess and it can be used for monitoring the response as well as ultrasonic guidance of aspiration [5]. In our patient this was done with optimal response. Treatment modalities like percutaneous

aspiration or drainage procedure with sonographic guidance, early surgical exploration and adjunctive antimicrobial therapy led to favourable outcome in preterm neonates [7].

Similarly, case series of 8 patients by Lee et al showed that percutaneous aspiration should be seriously considered in solitary liver abscess whenever possible, even in high risk patients because antibiotic alone may be insufficient and unsuccessful [8].

It is recommended that the duration of parenteral antibiotic therapy after drainage of liver abscess should be at least 2 weeks [9]. In our case timely adequate drainage and adjunctive antimicrobial therapy and collaboration with the interventional radiologist, Paediatric surgeon and neonatologist led to a favourable outcome in this preterm neonate.

\section{Funding: Nil}

\section{Conflict of interest: Nil}

Permission from IRB: Yes

\section{References}

1. Foster JH, History of liver surgery. J.Arch Surg. $1991 ; 126: 381-7$

2. Tan NWH, Sriram B, Tan-Kendrick APA, Rajadurai VS. Neonatal hepatic abscess in a preterm infants: A rare entity. Journal of Medicine Singapore.2005;34:558-64

3. Kutsunai T. Abscess of the liver of umbilical origin in infants: Report of 2 cases. Am J Dis Child 1936;51:13851396 
4. Bosnali O, Moralioglu S, Celayir A, Pektas O Liver abscess: Increasing occurrence in premature newborn Journal of Neonatal Surgery 2013;2(2):23

5. Moss TJ,Pysher TJ.Hepatic abscess in neonates.Am J Dis child 1981;135:726-728

6. Guillos B, Guillemin MG, Monnery JL, Alix D Neonatal pleuropulmonary staphylococcal infection with multiple abscesses of the liver[French] Ann Pediatr (Paris) 1989;36:681-84
7. Sharma S, Mohta A, Sharma P.Hepatic abscess in a preterm neonate Indian Pediatrics2007;44:226-7

8. Lee SH, Tomlinson C, Temple M, Amaral J, Connolly LB. Imaging -Guided Percutaneous Needle Aspiration or Catheter of Neonatal Liver Abscess:14- year Experience American Journal of Roentgenology.2008;190:616-622.

9. De franco PE, Shook LA, Goebel J, Lee B.Solitary liver abscess with associated glomerulonephritis in a neonate.J Perinato 1 2000;20:384-6

\section{How to cite this article?}

Anand S, Chanchlani R, Gupta V, Goyal S, Tiwari A. Hepatic Abscess in Preterm Newborn- A rare entity. Int J Med Res Rev 2014;2(2):169-171. doi:10.17511/ijmrr.2014.i02.20 